\title{
Influence of High Hydrostatic Pressure on the Flow Stress of Zinc and Zirconium Polycrystals*
}

\author{
By Atsushi Oguchi**, Susumu Yoshida** \\ and Minoru Nobuki**
}

\begin{abstract}
The influence of high hydrostatic pressure on the flow stress of zinc and zirconium polycrystals has been investigated at room temperature. Pressurizing under a pressure of $12000 \mathrm{~kg} / \mathrm{cm}^{2}$, tensile tests using the differential pressure method between atmospheric pressure and $12000 \mathrm{~kg} / \mathrm{cm}^{2}$, and tensile tests under a constant hydrostatic pressure of $12000 \mathrm{~kg} / \mathrm{cm}^{2}$ have been carried out. The tensile load has been measured by a magnetostrictive load cell in the high pressure chamber. The results obtained are as follows : (1) Both materials belong to the hexagonal close-packed system and are anisotropic in their elastic constants. Therefore, it is expected that shear stresses are generated at grain boundaries when they are subjected to high hydrostatic pressure and cause the pressurizing effect. The effect of pressurizing, however, is very small and is within the error of measurement for both materials at atmospheric pressure. The reason seems to be that for zinc the most part of workhardening caused by pressurizing disappears rapidly due to recovery before tensile test is carried out while for zirconium the shear strain caused by pressurizing is within the elastic region. But it is presumed that a pressurizing effect would remain latently under high hydrostatic pressure if the annealed material is not returned to atmospheric pressure. (2) In the differential pressure method, the change of flow stress with ambient pressure includes the latent pressurizing effect. At large strains where the latent pressurizing effect decreases and becomes negligibly small, the change of flow stress between $12000 \mathrm{~kg} / \mathrm{cm}^{2}$ and atmospheric pressure is shown to be about $8 \%$ for zinc and about $3 \%$ for zirconium. These values coincide with the variation in shear modulus of the materials with hydrostatic pressure which is estimated theoretically. (3) The work-hardening is enhanced for these materials under hydrostatic pressure. In the case of zirconium, the tendency of enhancement is similar to that observed with cubic metals. That is, at first it increases and then becomes saturated. For zinc, the enhancement continues to increase with increasing strain in the strain range tested. This seems to be due to the fact that recovery is suppressed under hydrostatic pressure, while it takes place actively at atmospheric pressure.
\end{abstract}

(Received June 3, 1971)

\section{Introduction}

The present authors have already reported on the influence of hydrostatic pressure on the flow stress of aluminium $^{(1)}$, copper ${ }^{(2)}$, iron and molybdenum ${ }^{(3)}$ polycrystals. The influence of hydrostatic pressure on the flow stress of metallic materials can, in general, be divided into three elements. The first is the so-called pressurizing effect, which is a kind of prestraining effect caused by shear stress components induced by hydrostatic pressure when the material contains discontinuities of compressibility in the matrix. This phenomenon was observed in tough pitch copper containing a large amount of $\mathrm{Cu}_{2} \mathrm{O}$ inclusions and pure iron with high impurity oxygen. The second is the influence of hydrostatic pressure on the process of workhardening. In all of the above four kinds of metals, the work-hardening was found to be enhanced. The third is the influence of hydrostatic pressure on the flow stress for a given work-hardened structure and it can be measured only by the differential pressure test. The change in flow stress $\Delta \sigma / \sigma$ due to the third element is mainly caused by the change of shear modulus $\Delta G / G$ under hydrostatic pressure. Moreover, there is another possibility of increase of flow stress when the thermally activated process which controls plastic deformation is accompanied by an increase of specific volume ${ }^{(4)(5)(6)}$.

* This paper was published in Japanese in J.Japan Inst. Metals, 35 (1971), 371.

** National Research Institute for Metals, Tokyo, Japan.

(1) S. Yoshida and A. Oguchi : Trans. JM, 11 (1970), 424.

(2) S. Yoshida, A. Oguchi and M. Nobuki : Trans. JIM, (in press).

Trans. J I M
Among the above four metals, the increase of flow stress attributable to this origin was observed in aluminium and the lower yield stress of pure iron.

Both zinc and zirconium taken up in this paper belong to the hexagonal close-packed metals and are anisotropic in their elastic properties, differing from the cubic metals studied in the previous work by the authors. The present paper describes results of investigations on the changes in flow stress of these metals under hydrostatic pressure and compares them with the results for the cubic metals.

\section{Materials and Experimental Procedures}

The specimens were prepared as follows. An ingot of pure zinc (99.99\%) was cold-rolled and drawn to a wire $2.5 \mathrm{~mm}$ in diameter. The wire was cut into tensile specimens and the specimens were at first left at room temperature for one day and then kept at about $-2{ }^{\circ} \mathrm{C}$ until they were tested. As for zirconium, commercial sponge zirconium (99\%) was melted in an electron beam melting furnace. The ingot was cold-worked to a wire $1.1 \mathrm{~mm}$ in diameter after annealing at $700^{\circ} \mathrm{C}$ for $2 \mathrm{hr}$, and then cut into tensile specimens and annealed in

(3) A. Oguchi, S. Yoshida and M. Nobuki : Trans. JIM, 13 (1972), 63.

(4) P. Haasen and A. W. Lawson : Z. Metallk., 49 (1958), 280.

(5) L.A. Davis and R.B. Gordon : J. Appl. Phys., 39 (1968), 3885.

(6) J.E. Hanafee and S. V. Radcliffe : J. Appl. Phys., 38(1967), 4284 .

1972 Vol. 13 
vacuum at $750^{\circ} \mathrm{C}$ for $2 \mathrm{hr}$. The mean grain diameter of annealed specimens was about $0.1 \mathrm{~mm}$ for zinc and about $0.03 \mathrm{~mm}$ for zirconium. In the present paper these specimens will be denoted as $\mathrm{Zn}$ and $\mathrm{Zr}$, respectively. The specimens were gripped mechanically at both ends and deformed in tension, the gauge length between the chucks being $15 \mathrm{~mm}$.

The experiments were carried out by the following three procedures: (1) A tensile test is done at atmospheric pressure for a specimen pressurized at 12000 $\mathrm{kg} / \mathrm{cm}^{2}$ and the flow stress is compared with that of the annealed specimen. (2) A tensile test is done under a constant pressure of $12000 \mathrm{~kg} / \mathrm{cm}^{2}$ and the flow stress is compared with that at atmospheric pressure. (3) After once pressurized at $12000 \mathrm{~kg} / \mathrm{cm}^{2}$, a specimen is pulled in tension under atmospheric pressure and 12000 $\mathrm{kg} / \mathrm{cm}^{2}$ alternately and the change of flow stress with pressure change is measured (the differential pressure method).

For the tensile tests under high hydrostatic pressure, the same apparatus as described in the previous papers $^{(1)(2)(7)}$ was used. In brief, by this apparatus tensile tests under constant hydrostatic pressures up to $15000 \mathrm{~kg} / \mathrm{cm}^{2}$ can be carried out. A magnetostrictive load cell ${ }^{(8)(9)}$ was used for the measurement of tensile loads under hydrostatic pressure. The zero shift and the variation of the gauge factor of this cell under pressure are very small, and particularly for the latter it has been established that the load-response relation under hydrostatic pressures up to $12000 \mathrm{~kg} / \mathrm{cm}^{2}$ scarcely differs from that at atmospheric pressure up to a load of $200 \mathrm{~kg}$.

Tests were carried out at a pulling rate of $0.5 \mathrm{~mm} / \mathrm{min}$ at room temperature.

\section{Experimental Results}

\section{Pressurizing effect}

Annealed specimens were kept for $10 \mathrm{~min}$ under a pressure of $12000 \mathrm{~kg} / \mathrm{cm}^{2}$ and deformed by tension at atmospheric pressure $10 \mathrm{~min}$ after pressure releasing. The results are shown in Fig. 1 . In the figure, the results for non-pressurized specimens are also shown. As obviously seen in the figure, the effect of pressurizing at this pressure on the load-elongation curve at atmospheric pressure was so small as to be within the scatter of data for both $\mathrm{Zn}$ and $\mathrm{Zr}$.

\section{Tensile testing by differential pressure method}

Figure 2 shows an example of the results of tensile tests by the differential pressure method between atmospheric pressure and $12000 \mathrm{~kg} / \mathrm{cm}^{2}$ for $\mathrm{Zr}$. In the case of $\mathrm{Zr}$, the transition point from elastic to plastic deformation, i.e., the bend due to yielding, on the loadelongation curve of reloading was clearly recognized.

(7) S. Yoshida and A. Oguchi : J. Soc. Mat. Sci. Japan, 18 (1969), 779 , (in Japanese).

(8) A. Oguchi and S. Yoshida : Japan J. Appl. Phys., 7 (1968), 672

(9) A. Oguchi : J. Japan Soc. Prec. Eng., 36 (1970), 474.

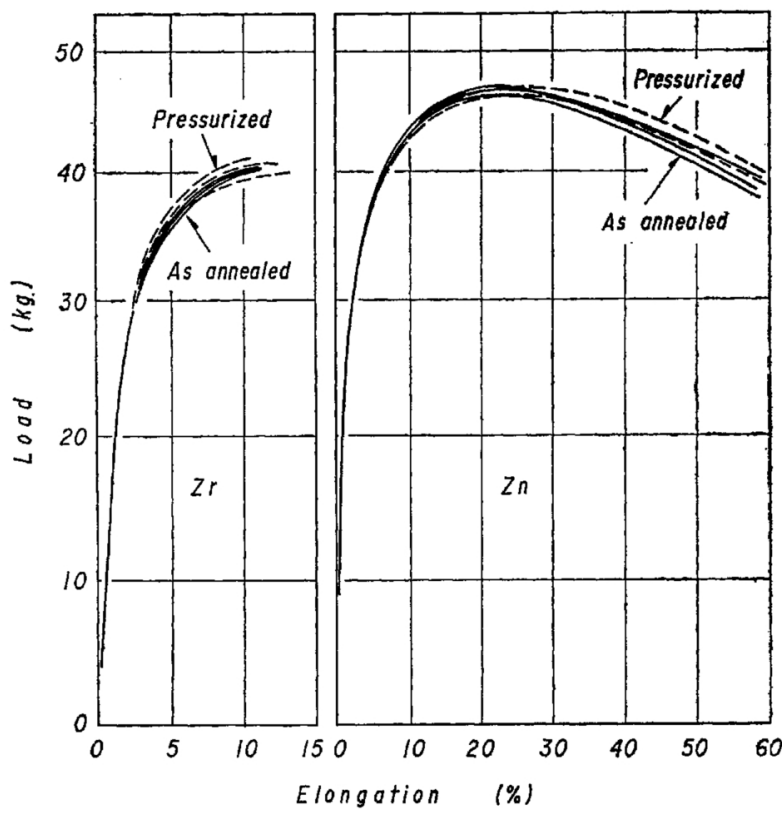

Fig. 1 Load-elongation relations for specimens pressurized under a pressure of $12000 \mathrm{~kg} / \mathrm{cm}^{2}$ and tensile-tested at atmospheric pressure. The same relations for annealed specimens are also shown for comparison.

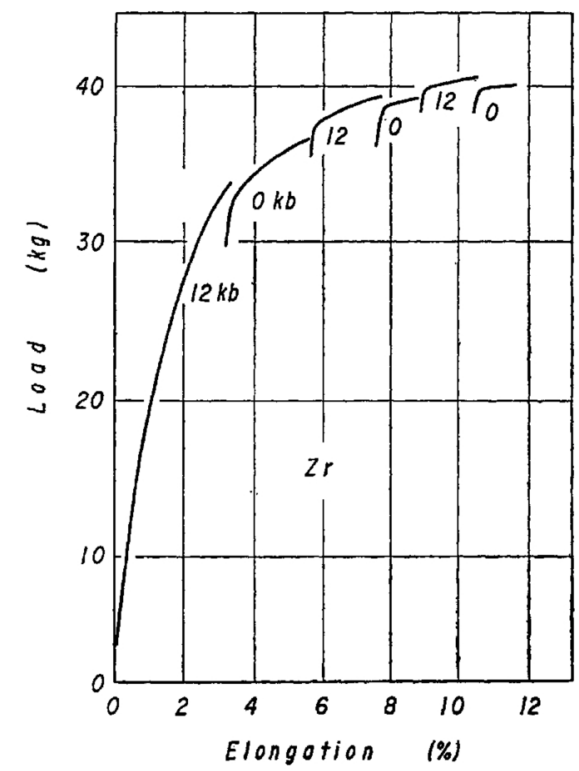

Fig. 2 An example of load-elongation relation for a polycrystalline specimen of zirconium obtained on changing the ambient pressure between atmospheric pressure and $12000 \mathrm{~kg} / \mathrm{cm}^{2}$.

Therefore, the point of bend on the load-elongation curve was taken as the tensile load on reloading. Figure 3 shows the change of flow stress due to pressure change obtained from the change of tensile load taking the contraction of the cross-section of the specimen under pressure into consideration. As shown in the figure, the change of flow stress due to hydrostatic pressure decreases with increasing strain and tends to become almost constant at about $7 \%$ strain.

As for $\mathrm{Zn}$, on the other hand, the result could not be treated as in the case of previous materials, because the decrease of tensile load due to interruption of deformation was very large as shown in Fig. 4 . Therefore, as schematically shown in Fig. 5 , the tensile load at $0.8 \%$ 


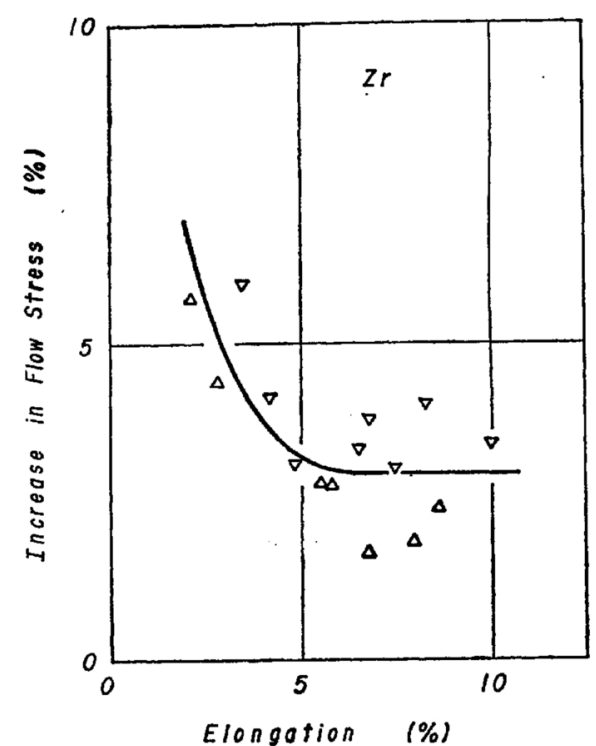

Fig. 3 Increase in flow stress of zirconium polycrystals due to a hydrostatic pressure of $12000 \mathrm{~kg} / \mathrm{cm}^{2}$ obtained from the differential pressure test. $\Delta$ : from atmospheric pressure to $12000 \mathrm{~kg} / \mathrm{cm}^{2}$ $\nabla:$ from $12000 \mathrm{~kg} / \mathrm{cm}^{2}$ to atmospheric pressure

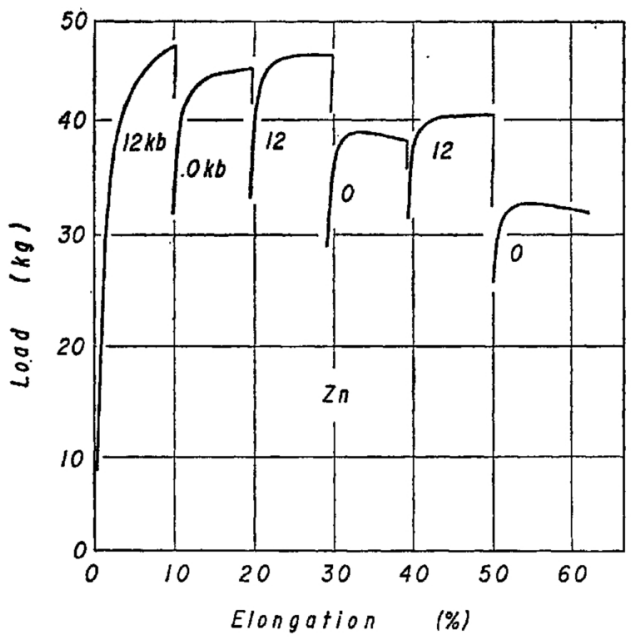

Fig. 4 An example of load-elongation relation for a polycrystalline specimen of zinc obtained on changing the ambient pressure between atmospheric pressure and $12000 \mathrm{~kg} / \mathrm{cm}^{2}$.

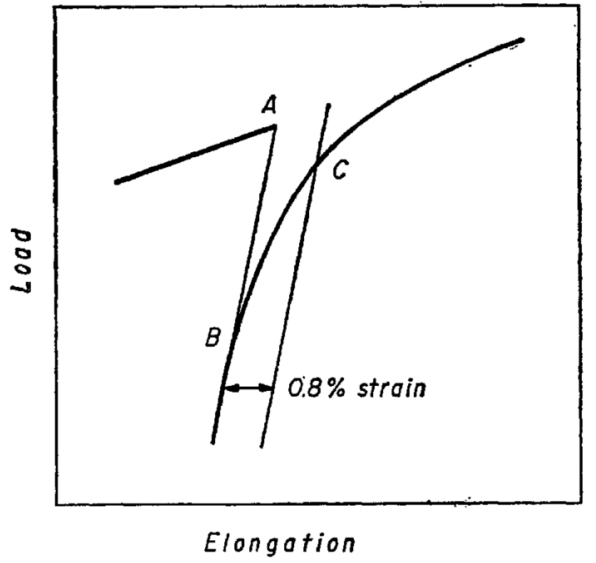

Fig. 5 Schematical representation of the effect of unloading during a differential pressure test for zinc. The tensile load $C$ is compared with the load A. permanent strain (point $\mathrm{C}$ ) was taken in place of that at the beginning of plastic deformation (point B) and compared with the load before pressure changing (point A). The change in the tensile load due to pressure change obtained by such an operation is shown in Fig. 6 . As shown in the figure, the difference of the change due to the direction of pressure change is very large so that they should not be simply averaged.

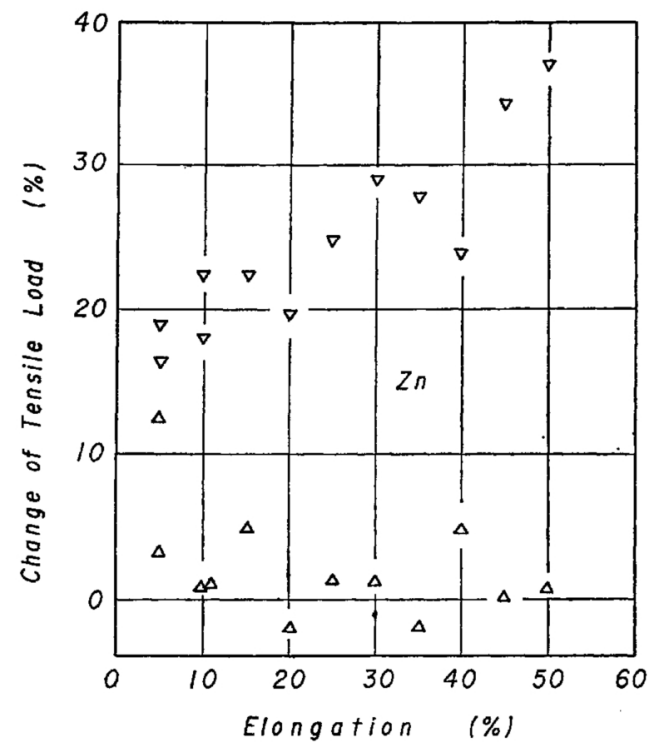

Fig. 6 Change of tensile load due to a hydrostatic pressure of $12000 \mathrm{~kg} / \mathrm{cm}^{2}$ obtained from the result of differential pressure tests for zinc using the method illustrated in Fig. 5.

$\triangle$ : from atmospheric pressure to $12000 \mathrm{~kg} / \mathrm{cm}^{2}$ $\nabla:$ from $12000 \mathrm{~kg} / \mathrm{cm}^{2}$ to atmospheric pressure

As Drouard et al. ${ }^{(10)}$ showed for single crystals of zinc prestrained at low temperatures, the decrease of flow stress due to recovery in zinc is very rapid at room temperature. About $10 \mathrm{~min}$ after unloading approximately $60 \%$ of work-hardening is recovered at $30^{\circ} \mathrm{C}$. Thus, the recovery which takes place during the period of unloading is very rapid even at room temperature in pure zinc and needs to be taken into consideration. Now, the process of recovery in the present experiment will be examined in detail. The deformation of a specimen under a pressure $P_{1}$ is interrupted at a certain strain. The specimen is unloaded and the ambient pressure is immediately changed to $P_{2}$. After about 10 min of unloading period, during which the temperature of the pressure medium returns to the original equilibrium value, the specimen is reloaded under $P_{2}$. That is, the greater part of recovery takes place under $P_{2}$ in this case. Consequently, the recovery during the unloading period on the way of tensile testing by the differential pressure method occurs at atmospheric pressure in the case of pressure releasing (from $12000 \mathrm{~kg} / \mathrm{cm}^{2}$ to atmospheric pressure) and under $12000 \mathrm{~kg} / \mathrm{cm}^{2}$ in the case of pressure raising. As the effect of recovery increases the change of the tensile load for pressure releasing and decreases that for pressure raising in the differential pressure test, the difference due to the

(10) R. Drouard, J. Washburn and E.R.Parker : J. Metals, 5 (1953), 1226. 
direction of pressure changing shown in Fig. 6 is considered to be caused by this effect.

On the other hand, it is well known that the selfdiffusion of metallic materials is influenced by hydrostatic pressure. Therefore, the rate or the amount of recovery is presumed to be influenced by the ambient pressure. Thus, it is considered that the effect of recovery cannot be eliminated simply by taking the average of change of load for pressure raising and releasing. In order to examine the influence of pressure on the recovery of zinc, a specimen was pulled under $12000 \mathrm{~kg} / \mathrm{cm}^{2}$ and unloaded during the test at various strains and subjected to recovery under $12000 \mathrm{~kg} / \mathrm{cm}^{2}$ or at atmospheric pressure for $10 \mathrm{~min}$. Then, the specimen is reloaded under $12000 \mathrm{~kg} / \mathrm{cm}^{2}$. An example of load-elongation curve obtained by this test is shown in Fig. 7. From such curves the decrease in the flow stress due to recovery were obtained by the method represented in Fig. 5 and are shown in Fig. 8. As the method of Fig. 5 was adopted for convenience' sake, the

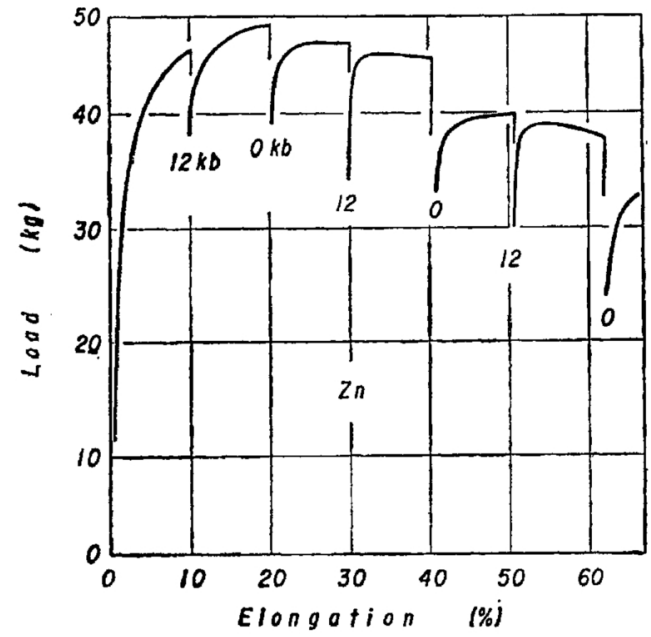

Fig.7 An example of load-elongation relation for a polycrystalline specimen of zinc tested under a pressure of $12000 \mathrm{~kg} / \mathrm{cm}^{2}$. The test is interrupted at intervals and unloaded for $10 \mathrm{~min}$ at atmospheric pressure or under $12000 \mathrm{~kg} / \mathrm{cm}^{2}$ before reloading.

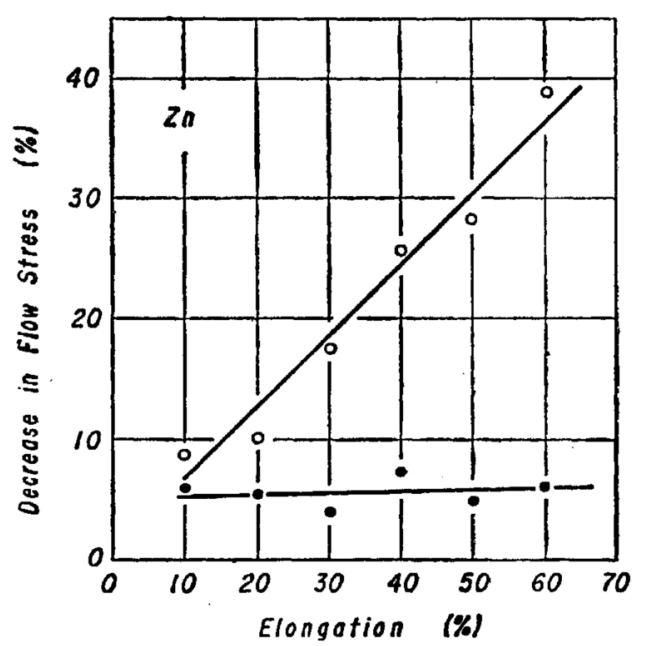

Fig. 8 Decrease in flow stress due to unloading of $10 \mathrm{~min}$ under pressure of $12000 \mathrm{~kg} / \mathrm{cm}^{2}$ (solid circles) or at atmospheric pressure (open circles) obtained from Fig. 7 using the method illustrated in Fig. 5. result shown in Fig. 8 cannot be considered to represent the amount of recovery itself, but it can be said that the amount of recovery is very large at atmospheric pressure as compared with that under $12000 \mathrm{~kg} / \mathrm{cm}^{2}$ and its rate increases with increasing strain. Using the result of Fig. 8, the effect of recovery included in the result of Fig. 6 can be eliminated. In Fig. 9, the result after such correction is made is shown. Here, the change of cross-section of the specimen by hydrostatic pressure is taken into account.

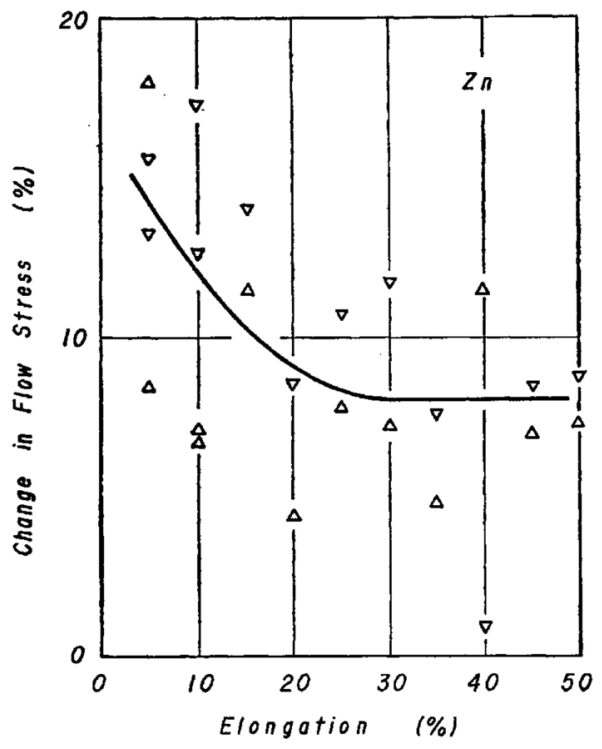

Fig. 9 Increase in flow stress of zinc polycrystals due to a hydrostatic pressure of $12000 \mathrm{~kg} / \mathrm{cm}^{2}$ obtained from the result of Fig. 6 corrected for the effect of unloading using the data of Fig. 8 .

$\Delta:$ from atmospheric pressure to $12000 \mathrm{~kg} / \mathrm{cm}^{2}$ $\nabla:$ from $12000 \mathrm{~kg} / \mathrm{cm}^{2}$ to atmospheric pressure

As shown in the figure, the change of the flow stress is very large at small strains, decreases with increasing strain, and becomes almost constant (about 8\%).

\section{Tensile test under a constant hydrostatic pressure}

Figure 10 shows the increase of the tensile load under $12000 \mathrm{~kg} / \mathrm{cm}^{2}$ as compared with that at atmospheric pressure at the same values of strain. In the same figure, the change of the flow stress is also shown by dotted lines. As shown in the figure, the change for $\mathrm{Zn}$ is large at small strains and shows a minimum value at about $20 \%$ strain, and then increases with increasing strain. For $\mathrm{Zr}$, on the other hand, the change is almost constant showing no dependence on strain. The value of the increase in the flow stress is about $11 \%$ at about $20 \%$ strain for $\mathrm{Zn}$ and about $8.4 \%$ for $\mathrm{Zr}$. In the present experiment for $\mathrm{Zn}$, the local necking of the specimen did not take place beyond the maximum point of load-elongation curve and the uniform deformation continued up to about $50 \%$ strain. Therefore, the scope of the present investigation was limited up to this strain. 

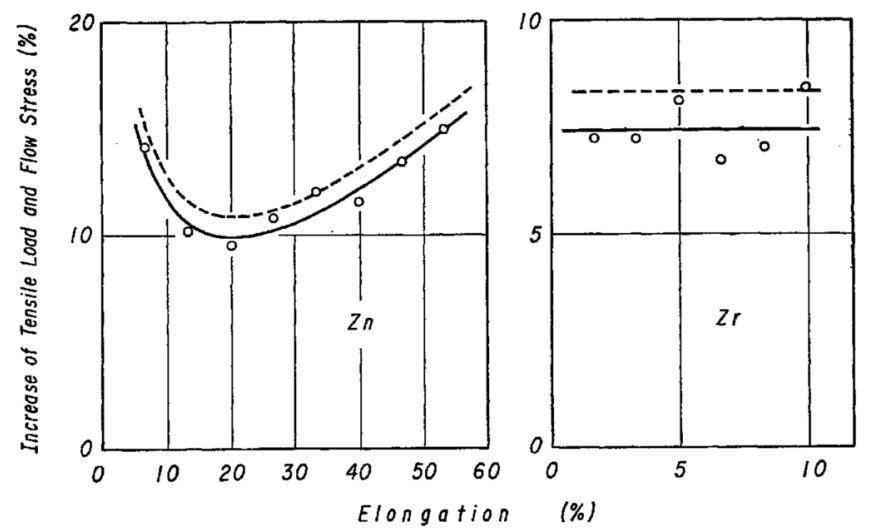

Fig. 10 Increase of tensile load (solid line) and flow stress (dotted line) under a pressure of $12000 \mathrm{~kg} / \mathrm{cm}^{2}$ as compared with that at atmospheric pressure.

\section{Discussion}

\section{Pressurizing effect}

As already discussed by many investigators, the pressurizing effect is considered to originate from elastic discontinuities in materials. In metals of the cubic system, the existence of inclusions which are different in compressibility from the matrix is expected to be the cause of this effect. Therefore, it will not be observed in single phase metals. On the other hand, for metals of the hexagonal close-packed system, grain boundaries are a possible cause of the pressurizing effect even in single phase metals because the linear compressibility depends, in general, largely on the crystallographic orientation. In this case, the problem to be considered is the difference in the linear compressibility across a grain boundary. The greater the difference is, the greater the induced shear stress components. According to the existing data, the ratio of the linear compressibility on the c-axis to that on a-axis $\left(\kappa_{c} / \kappa_{a}\right)$ is about $7^{(11)}$ for zinc and about $0.85^{(12)}$ for zirconium. Therefore, the shear stress components are induced at grain boundaries under hydrostatic pressure for these materials if they are polycrystalline. The induced shear stress is presumed to be much greater for zinc than those for zirconium. When the shear stress components are above the critical shear stress of the material, the plastic deformation will take place and the pressurizing effect will occur.

Annealed polycrystalline specimens were pressurized at $12000 \mathrm{~kg} / \mathrm{cm}^{2}$ for the purpose of examining for the occurrence of plastic deformation by pressurizing. The surfaces of both materials were polished flat chemically so as to avoid plastic deformation and after ensuring that there were neither slip bands nor deformation twins, the specimens were pressurized at $12000 \mathrm{~kg} / \mathrm{cm}^{2}$. The microscopic examination immediately performed after pressurizing revealed many slip bands and deformation twins on $\mathrm{Zn}$ specimens as shown in Photo. 1, while for $\mathrm{Zr}$ no deformation mark was observed.

From the above results, it may be concluded that for $\mathrm{Zr}$

(11) B. Jaoul : Etude de la Plasticité et Application aux Métaux, Dunod, Paris, (1965). p. 101.

(12) E. S. Fisher and C. J. Renken : J. Nuc. Mat., 4 (1961), 311.

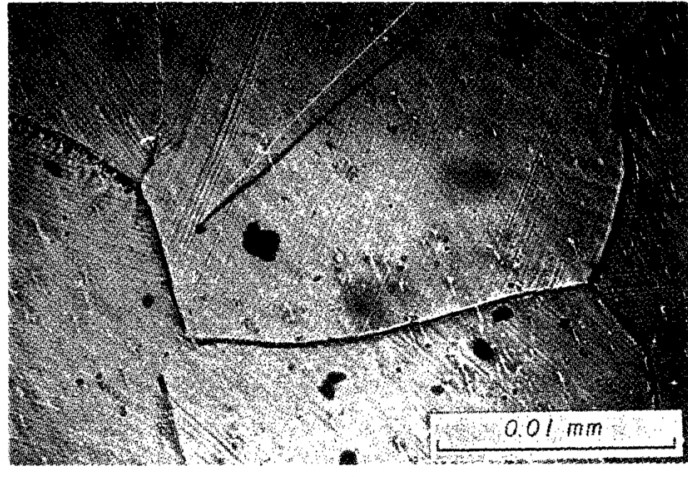

Photo. 1 Photomicrograph of slip bands and a twin observed on a zinc polycrystal pressurized under a pressure of $12000 \mathrm{~kg} / \mathrm{cm}^{2}$.

the shear stress components induced at grain boundaries are smaller than the critical shear stress as the value of $\kappa_{c} / \kappa_{a}$ is near unity as mentioned before and the shear strain induced by hydrostatic pressure remains within the region of elastic strain. Consequently, when the specimen of $\mathrm{Zr}$ is returned to atmospheric pressure, its internal state goes completely back to the original one and the effect of pressurizing is not observed on tensile testing at atmospheric pressure. On the other hand, the value of $\kappa_{c} / \kappa_{a}$ is so large and the critical shear stress for slip is so small for $\mathrm{Zn}$ that slip bands and deformation twins are induced by pressurizing as shown in Photo. 1. Since the rate of recovery in this material is very large at atmospheric pressure even at room temperature as mentioned before, the greater part of work-hardening recovers during the period between de-pressurizing and tensile testing and consequently the so-called pressurizing effect is not observed.

As discussed above, it can be understood that for both $\mathrm{Zn}$ and $\mathrm{Zr}$ the so-called pressurizing effect cannot be observed by the conventional experimental procedure in which the annealed specimen is deformed at atmospheric pressure after being pressurized. But, if the specimens are held as they are under hydrostatic pressure, the shear stress components will remain at grain boundaries in $\mathrm{Zr}$, while slip will be generated from grain boundaries and the work-hardened state induced by them will be kept in $\mathrm{Zn}$ because of the small rate of recovery under high hydrostatic pressure. Namely, if the specimens are brought back to atmospheric pressure, the pressurizing effect cannot be observed in both materials, while the effect will remain if the specimens are continuously deformed under hydrostatic pressure. But, the pressurizing effect in this case is inevitably mixed with the other effect as will be discussed later in Section 2 and cannot be measured separately as the conventional pressurizing effect. For such a reason, this effect should be called "latent pressurizing effect" as distinguished from the conventional one, but it will be called as pressurizing effect in the following discussion for the sake of simplicity.

\section{Effect of hydrostatic pressure on the flow stress of material with a given internal structure}

As the temperature of recrystallization or recovery in 
$\mathrm{Zr}$ is very high as compared with that in $\mathrm{Zn}$, the decrease of flow stress due to recovery during the unloaded period for pressure changing is thought to be very small in $\mathrm{Zr}$. Therefore, the result in Fig. 3 shows the change of the flow stress for a given internal structure by the pressure change. On the other hand, the internal structure of a specimen of $\mathrm{Zn}$ cannot be considered to be invariable throughout pressure change because of its large rate of recovery as mentioned before. But, the effect of recovery has been corrected and eliminated in the result of Fig. 9.

For both $\mathrm{Zn}$ and $\mathrm{Zr}$ there exists the latent pressurizing effect as discussed in the foregoing paragraph. The change of the flow stress for $\mathrm{Zr}$ shown in Fig. 3 includes the change due to this pressurizing effect. Also for $\mathrm{Zn}$ it is seen from the process of correction of recovery that the pressurizing effect is included in the change of the flow stress in Fig. 9.

As for the relation between the strain and the increase of the flow stress due to pressurizing measured at atmospheric pressure, the rate of increase is very large at the beginning of deformation and decreases with increasing strain, and then vanishes as in the case of tough pitch copper. The same tendency is expected for $\mathrm{Zn}$ and $\mathrm{Zr}$, and moreover, the latent pressurizing effect, too, will decrease with increasing strain owing to the decrease of misorientation between grains by the rotation of grains with deformation. Anyway, interpreting that the increase of the change in the flow stress observed at small strains in Figs. 3 and 9 is due to the pressurizing effect, the final value of the change at large strains (about $8 \%$ for $\mathrm{Zn}$ and about $3 \%$ for $\mathrm{Zr}$ ) is thought to represent the effect of hydrostatic pressure on the flow stress of the material with a given internal structure.

As discussed in the previous papers, the effect of hydrostatic pressure on the flow stress of a material with a given internal structure is represented as

$$
\frac{\Delta \sigma}{\sigma}=\frac{\Delta G}{G}+\Phi
$$

The first term in the right-hand side is the change of the shear modulus $G$ by hydrostatic pressure and the second term represents the increase of the flow stress which corresponds to an extra work to be applied to the material under hydrostatic pressure when the specific volume increases with deformation.

The value of $\Delta G / G$ by hydrostatic pressure for $\mathrm{Zn}$ has not yet been obtained experimentally, but it can be estimated theoretically by the following way. That is, the relation ${ }^{(13)}$

$$
\left(\frac{\partial \ln G}{\partial \ln V}\right)_{T}=-\left(2 \gamma+\frac{1}{3}\right)
$$

may be used, where $V$ is the specific volume, $r$ is the so-called Grüneisen constant which is represented as

$$
\gamma=\frac{\beta}{\chi C_{V}}
$$

(13) R. W. Keys : Solids under Pressure, McGraw-Hill Book Co., (1963), p. 77 . where $\beta$ is the coefficient of thermal expansion, $\chi$ is the compressibility and $C_{V}$ is the specific heat per unit volume. From eq. (2) we obtain

$$
\begin{aligned}
\left(\frac{\partial \ln G}{\partial \ln V}\right)_{T} & =\frac{\Delta G}{G} \frac{V}{\Delta V}=\frac{1}{\chi P} \frac{\Delta G}{G} \\
& =-\left(2 \gamma+\frac{1}{3}\right) .
\end{aligned}
$$

Then, using the value of $\gamma$ we can calculate $\Delta G / G$ under $12000 \mathrm{~kg} / \mathrm{cm}^{2}$. The results are shown in Table 1 . As the experiments were carried out for polycrystals, the above calculation was done by averaging the difference of physical properties due to the crystallographic orientation.

Table 1 Change of shear modulus and flow stress due to hydrostatic pressure (\%).

\begin{tabular}{c|c|c|c|c|c}
\hline \hline & $\gamma$ & $2 \gamma+{ }_{3}^{1}$ & $\left(\frac{\Delta G}{G}\right)_{\text {cal. }}^{12 \mathrm{~kb}}$ & $\left(\frac{\Delta \sigma}{\sigma}\right)_{\text {exp }}^{12 \mathrm{~kb}}$ & $\left(\frac{\Delta G}{G}\right)_{\text {exp. }}^{12 \mathrm{~kb}}$ \\
\hline Zinc & 1.90 & 4.13 & 8.4 & 8 & - \\
Zirconium & 0.84 & 2.01 & 2.5 & 2.9 & $-0.24^{(14)}$ \\
\hline \hline
\end{tabular}

As shown in the table, the calculated values of $\Delta G / G$ agree fairly well with the experimental values of $\Delta \sigma / \sigma$ for both $\mathrm{Zn}$ and $\mathrm{Zr}$. That is, if the calculated values of $\Delta G / G$ are correct, the increase of $\sigma$ can be explained solely by the increase of shear modulus by hydrostatic pressure. But the experimental value of $\Delta G / G$ by hydrostatic pressure for zirconium obtained by Bridgman $^{(14)}$ is largely different from the calculated value as shown in the table. Concerning this point, it seems necessary to examine experimentally the change of shear modulus under hydrostatic pressure once again. As the specimen was a coil of drawn wire of zirconium and might have had a preferential fibre structure in the experiment by Bridgman, it is supposed that his result was influenced by the anisotropy of the specimen. Anyway, as the validity of eq. (2) is not so certain, the change of $\Delta G / G$ by hydrostatic pressure must be measured more exactly in future.

\section{Effect of hydrostatic pressure on work-hardening}

As mentioned in the foregoing paragraph, the flow stress increases at the beginning of deformation by the pressurizing effect for both $\mathrm{Zn}$ and $\mathrm{Zr}$ when the specimens are deformed under hydrostatic pressure. As for $\mathrm{Zn}$ in Fig. 10, the phenomenon that the increase of the flow stress is large at the early stage of deformation and then decreases gradually is thought to be due to the pressurizing effect. In the case of $\mathrm{Zr}$, however, the increase of the flow stress appears to be constant in the strain range tested. This may be interpreted as follows: As the change of the flow stress due to the effect of hydrostatic pressure on work-hardening is presumed to increase starting from zero at the beginning of deformation, the decrease of the pressurizing effect is compensated by this increase.

(14) P.W. Bridgman : The Physics of High Pressure, G. Bell and Sons, (1952), p. 386. 

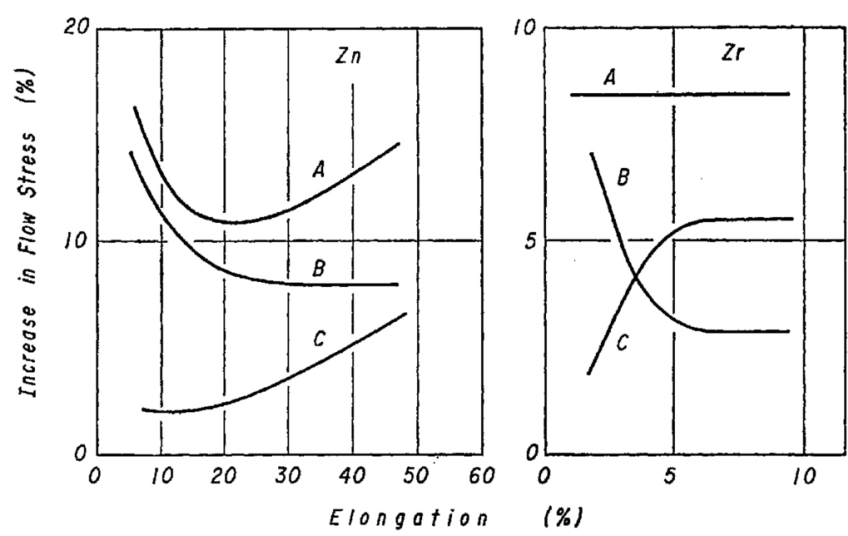

Fig. 11 Increase in flow stress of the materials on tensile testing under hydrostatic pressure as decomposed into the components which have different origins.

A : total increase, $B$ : the effect of pressure on the flow stress which includes the effect of pressurizing, $C$ : the effect of pressure on the work-hardening.

As mentioned before, $\Delta \sigma / \sigma$ shown in Figs. 3 and 9 includes the pressurizing effect. Consequently, by subtracting the results in Figs. 3 and 9 from those in Fig. 10, the change of work-hardening by hydrostatic pressure with increasing strain can be obtained. But, the pressurizing effect shown in Fig. 10 was given immediately before the deformation under hydrostatic pressure starts, while that in Figs. 3 and 9 was given by pressure raising after some amount of deformation at atmospheric pressure. Therefore, the physical meanings of the two cases are slightly different, and consequently the behaviour of the change of the flow stress against strain may also be a little different in detail. But the above method will be useful to understand the general trend.

Figure 11 shows the result of such analysis carried out graphically. As shown in the figure, for $\mathrm{Zr}$, the change in work-hardening increases with increasing strain and becomes almost constant at about $7 \%$ strain. As for $\mathrm{Zn}$, it continues to increase with increasing strain in the strain range tested. The tendency observed for $\mathrm{Zr}$ is similar to that observed previously with cubic metals. The fact that the tendency for $\mathrm{Zn}$ is different can be explained in such a way that the recovery in $\mathrm{Zn}$ takes place actively at atmospheric pressure even with deformation, while it is much suppressed under hydrostatic pressure.

\section{Conclusions}

The influence of hydrostatic pressure on the flow stress of zinc and zirconium polycrystals has been studied. The results obtained are as follows:

(1) Both materials belong to the hexagonal closepacked system and are anisotropic in their elastic constants. Therefore, it is expected that shear stresses are generated at grain boundaries when they are subjected to high hydrostatic pressure and cause the pressurizing effect. The effect of pressurizing, however, is very small and is within the error of measurement for both materials at atmospheric pressure. The reason seems to be that for zinc the most part of work-hardening caused by pressurizing disappears rapidly at atmospheric pressure due to recovery before tensile test is carried out while for zirconium the shear strain caused by pressurizing is within the elastic region. But it is presumed that a pressurizing effect will remain latently under high hydrostatic pressure if the annealed material is not returned to atmospheric pressure.

(2) As for the result by the differential pressure method, the change of flow stress with the ambient pressure includes the latent pressurizing effect. At large strains where the latent pressurizing effect decreases and becomes negligibly small, the difference of flow stress between $12000 \mathrm{~kg} / \mathrm{cm}^{2}$ and atmospheric pressure is shown to be about $8 \%$ for zinc and about $3 \%$ for zirconium. These values agree with the rates of change of whear modulus of the materials by hydrostatic pressure which are estimated theoretically.

(3) The work-hardening is enhanced for these materials under hydrostatic pressure. In the case of zirconium, the tendency of enhancement is similar to that observed with cubic metals. Namely, at first it increases and then becomes saturated. For zinc, the enhancement continues to increase with increasing strain in the strain range tested. This seems to be due to the fact that for zinc recovery is suppressed under hydrostatic pressure, while it takes place actively at atmospheric pressure. 